\title{
Line $\times$ Tester Analysis to Determine Extent of Heterosis for Various Yield and Quality Parameters in Sugarcane (Saccharum officinarum)
}

\author{
Deepankar Pandey*, S.P. Singh, A.S. Jeena and Tabassum
}

Department of Genetics and Plant Breeding, College of Agriculture, Govind Ballabh Pant

University of Agriculture and Technology, Pantnagar, U.S. Nagar, 263145, Uttarakhand, India

*Corresponding author

\begin{tabular}{|l|}
\hline Ke y w o r d s \\
$\begin{array}{l}\text { Sugarcane, Line x } \\
\text { tester, Hybrid } \\
\text { vigour, Economic } \\
\text { heterosis }\end{array}$ \\
\hline Article Info \\
\hline $\begin{array}{l}\text { Accepted: } \\
\text { 12 February } 2019 \\
\text { Available Online: } \\
\text { 10 March } 2019\end{array}$ \\
\hline
\end{tabular}

\section{Introduction}

Sugarcane cultivation dates back to the Vedic period and the earliest reference is found in
Indian writings of the period 1400 to 1000 years BC. Sugarcane is mainly grown in tropical and sub-tropical regions. Being a member of the grass family, it belongs to the 
genus Saccharum, tribe Andropogoneae, family Poaceae and characterized by high degree of polyploidy. Sugarcane is valuable mainly because of its ability to store high concentrations of sucrose, or sugar, in the stem and more recently for the production of ethanol, which is an important renewable biofuel source (Mennosi et al., 2008 and De Costa et al., 2011). Saccharum officinarum, Saccharum baeberi and Saccharum sinense are three cultivated species and Saccharum spontaneum and Saccharum robustum are two wild species of sugarcane. Saccharum officinarum is the most widely cultivated species of sugarcane. India is the second largest producer of sugarcane in the world after Brazil. Across the world, 70Percent sugar is manufactured from sugarcane and it is a major source of raw material for sugar industries and other allied group of by product industries. It is grown in 5.34 million hectare with total production of 345.6 Million tones and productivity of 64.7 tonnes/ha (Indian Sugar, 2014).

The study of the characters of agronomic and commercial interest in the progeny resulting from the crossings in sugarcanes is of great importance. This is because parents can be identified for hybridization program (Tyagi and Lal, 2005). Line $x$ Tester analysis is one of the methods used to identify genetic worth of material and to select the parents for hybridization. The line $\mathrm{x}$ tester mating scheme involves " $l$ " lines and " $t$ " testers. All the " $l$ " lines are crossed to each of " $t$ " testers and " $l$ " $\mathrm{x}$ " $t$ " full sib progenies produced. These progenies resulted from line $\mathrm{x}$ tester matings, along with or without the parents, can be tested in a replicated trial using suitable field design (Singh and Chaudhary 1985; Comstock and Robinson 1948). The genetic variability for the different traits studied in the hybrid experiments is important to the breeders. It means that there is a possibility of genetically improving the germplasm further through selections for the significant traits (Pswarayi and Vivek, 2008). There is also an opportunity to identify best parents and progenies among the experimental materials for the development of new hybrids and improvement programme. The genetic variability present in the present day sugarcane cultivars has hybrid origin. The Saccharum officinarum has been contributing for genetic variability in sugarcane more than $S$. spontaneum, $S$. sinense and S. barberi (Patil and Patel, 2017). Nowadays, main objective of a sugarcane breeding program is to obtain new cultivars having more productivity and improved industrial characteristics. Commercially cultivated sugarcane varieties are heterozygous and complex polyploids resulted in generation of great amount of genetic variability. The study of the characters of agronomic and commercial interest in the progeny resulting from the crossing in sugarcanes is of great importance. Shull (1952) defined heterosis as "the interpretation of increased vigour, size, fruitfulness, speed of development, resistance to disease and insect pests, or climatic rigors of any kind, manifested by crossbred organisms as compared with corresponding inbreds, as the specific results of unlikeness in the constitution of the uniting parental gametes". In sugarcane, there is a good scope for exploitation of hybrid vigour as it is vegetatively propagated crop (Verma and Singh 2004). The magnitude of heterosis provides a basis for determining genetic diversity and also serves as a guide to the choice of desirable parents (Loganathan et al., 2001). It is a measure of the superior performance of hybrids over mid parent (relative heterosis), over batter parent (heterobeltiosis), over check parent (economic heterosis) and is a mean of identifying superior genotypes. Therefore, present investigation was conducted to identify superior sugarcane cross combinations for better cane yield, sugar yield and its attributes 
through the expression of heterosis for different morphological and quality traits.

\section{Materials and Methods}

The mating plan involves crossing of four lines namely, CoPant 84212, CoPant 98224, CoPant 99213, CoPant 94213 with two testers which are CoPant 97222 and CoSe 92423, in line $\mathrm{x}$ tester mating design to produce eight full sib progenies. The crosses for the investigation were made in National Hybridization Garden at Sugarcane Breeding Institute; Coimbatore Tamil Nadu. These eight progenies along with the six parents and six check varities viz., Co 1148, Co J 64, Co S 8436, Co S 767, CoPant 3220 and Co 0238 were tested in randomised block design with four replications at the Sugarcane Breeding Experimental Block of Norman Borlaug Crop Research Centre, Govind Ballabh Pant University of Agriculture and Technology, Pantnagar, U. S. Nagar, Uttarakhand during 2013-2017. The biometrical observations were recorded for eight morphological characters viz., Germination percent, Number of Tillers/h, Number of Millable Canes/h, Cane thickness, Cane height, Single Cane weight, Cane yield/h, Commercial cane sugar (CCS) yield/h and five quality characters viz., Juice
Polarity Value, Juice Brix percent, Juice sucrose percent, Juice purity percent and Commercial cane sugar percent (CCS Percent). To test the significance of differences between treatments, analysis of variance was done as suggested Gomez and Gomez (1984). Heterosis effects were calculated as reported by Hayman (1958). The magnitude of heterosis was estimated in relation to respective mid parent (MP), better parent $(\mathrm{BP})$ and check parent $(\mathrm{CP})$.

\section{Results and Discussion}

The analysis of variance revealed that estimates of mean squares were found significant for all the characters except purity Percent indicating the presence of considerable diversity in the material under study (Table 1). The results obtained from the analysis of variance revealed high significant differences for characters viz., Germination Percent $(44.791 * *)$, Number of Tillers $\left(227.307^{* *}\right)$ Number of Millable Canes $(117.319 * *)$, Single Cane Weight $(0.033 * *)$, Brix Percent (8.289**), Pol Value (111.812**), Sucrose Percent $(5.597 * *)$, C.C.S. Percent (4.503**), and C.C.S. yield $(5.821 * *)$.

Table.1 Analysis of variance (mean squares) for different morphological and quality characters in sugarcane

\begin{tabular}{|l|l|c|c|c|}
\hline S.No. & Characters & \multicolumn{3}{|c|}{ Mean Squares } \\
\hline $\mathbf{1}$ & & Replication (d.f.=3) & Treatment (d.f.=13) & Error (d.f.=39) \\
\hline $\mathbf{2}$ & Germination Percent & 8.069 & $44.791^{* *}$ & 5.309 \\
\hline $\mathbf{3}$ & Tillers (000/h) & 9.645 & $227.307^{* *}$ & 36.020 \\
\hline $\mathbf{4}$ & NMC (000/h) & 34.962 & $117.319^{* *}$ & 18.341 \\
\hline $\mathbf{5}$ & Height & 0.037 & $0.087^{*}$ & 0.041 \\
\hline $\mathbf{6}$ & Diameter & 0.061 & $0.137^{*}$ & 0.561 \\
\hline $\mathbf{7}$ & Single cane weight & 0.003 & $0.033^{* *}$ & 0.011 \\
\hline $\mathbf{8}$ & Brix(2015) & 1.445 & $8.289^{* *}$ & 1.018 \\
\hline $\mathbf{9}$ & Pol(2015) & 29.757 & $111.812^{* *}$ & 15.438 \\
\hline $\mathbf{1 0}$ & Sugar & 1.344 & $5.597^{* *}$ & 0.793 \\
\hline $\mathbf{1 1}$ & Purity Percent & 0.270 & 4.997 & 2.924 \\
\hline $\mathbf{1 2}$ & CCS Percent & 0.672 & $4.503^{* *}$ & 0.397 \\
\hline $\mathbf{1 3}$ & Cane yield & 141.687 & $136.437^{*}$ & 88.282 \\
\hline
\end{tabular}


Table.2 Estimation of heterosis for different characters

\begin{tabular}{|c|c|c|c|c|c|c|c|c|c|}
\hline \multirow[t]{3}{*}{ S.No. } & \multirow[t]{3}{*}{ CROSSES } & \multicolumn{8}{|c|}{ 1. Germination percent } \\
\hline & & \multirow[t]{2}{*}{ Relative heterosis } & \multirow[t]{2}{*}{ Heterobeltiosis } & \multicolumn{6}{|c|}{ Standard heterosis } \\
\hline & & & & Co 1148 & Co J 64 & Co S 8436 & CoPant 3220 & Co 0238 & Co S 767 \\
\hline 1. & CoPant $84212 \times$ CoPant 97222 & -0.22 & -2.62 & $11.93 *$ & $20.56 * *$ & $16.86 * *$ & $23.90 * *$ & $14.15 *$ & $18.73 * *$ \\
\hline 2. & CoPant $84212 \times$ CoSe 92423 & $-31.57 * *$ & $-31.76 * *$ & $-21.12 * *$ & $-15.04 *$ & $-17.64 * *$ & $-12.69 *$ & $-19.56 * *$ & $-16.33 * *$ \\
\hline 3. & CoPant $98224 \times$ CoPant 97222 & -5.01 & $-10.42 *$ & -1.99 & 5.57 & 2.33 & 8.49 & -0.05 & 3.96 \\
\hline 4. & CoPant $98224 \times$ CoSe 92423 & $-19.34 * *$ & $-25.85 * *$ & $-14.28 *$ & -7.67 & -10.50 & -5.12 & $-12.59 *$ & -9.08 \\
\hline 5. & CoPant $99213 \times$ CoPant 97222 & -7.25 & $-10.57 *$ & -2.15 & 5.39 & 2.16 & 8.31 & -0.22 & 3.79 \\
\hline 6. & CoPant $99213 \times$ CoSe 92423 & 0.37 & -5.70 & 9.00 & $17.40 * *$ & $13.80 *$ & $20.65 * *$ & 11.16 & $15.62 *$ \\
\hline 7. & CoPant $94213 \times$ CoPant 97222 & $-13.88 * *$ & $-15.03 * *$ & -7.03 & 0.13 & -2.94 & 2.90 & -5.20 & -1.39 \\
\hline \multirow[t]{2}{*}{8.} & CoPant $94213 \times$ CoSe 92423 & $-13.71 * *$ & $-17.11 * *$ & -4.18 & 3.20 & 0.03 & 6.06 & -2.29 & 1.63 \\
\hline & & \multicolumn{8}{|c|}{ 2. Tillers $(000 / \mathrm{h})$} \\
\hline 1. & CoPant $84212 \times$ CoPant 97222 & $13.75 * *$ & $11.01 * *$ & $17.19 * *$ & $13.90 * *$ & $8.82 *$ & $21.73 * *$ & $12.35 * *$ & $13.82 * *$ \\
\hline 2. & CoPant $84212 \times$ CoSe 92423 & $-10.48 * *$ & $-15.22 * *$ & -4.72 & -7.40 & $-11.52 * *$ & -1.02 & $-8.65 *$ & -7.46 \\
\hline 3. & CoPant $98224 \times$ CoPant 97222 & $6.76 *$ & 5.88 & $11.77 * *$ & $8.63 *$ & 3.79 & $16.10 * *$ & 7.16 & $8.56 *$ \\
\hline 4. & CoPant $98224 \times$ CoSe 92423 & -3.35 & -7.04 & 4.48 & 1.55 & -2.97 & $8.53 *$ & 0.17 & 1.48 \\
\hline 5. & CoPant $99213 \times$ CoPant 97222 & 3.71 & -0.93 & $14.86 * *$ & $11.63 * *$ & 6.66 & $19.31 * *$ & $10.12 *$ & $11.56 * *$ \\
\hline 6. & CoPant $99213 \times$ CoSe 92423 & -3.49 & -4.97 & $10.18 *$ & 7.08 & 2.31 & $14.45 * *$ & 5.63 & 7.01 \\
\hline 7. & CoPant $94213 \times$ CoPant 97222 & -0.53 & -0.92 & 4.60 & 1.66 & -2.87 & $8.65 *$ & 0.28 & 1.59 \\
\hline \multirow[t]{2}{*}{8.} & CoPant $94213 \times$ CoSe 92423 & $-11.17 * *$ & $-14.20 * *$ & -3.57 & -6.28 & $-10.45 * *$ & 0.17 & -7.55 & -6.34 \\
\hline & & \multicolumn{8}{|c|}{ 3. N.M.C. 000/h } \\
\hline 1. & CoPant $84212 \times$ CoPant 97222 & 5.61 & 5.33 & -1.30 & $7.85 *$ & -1.87 & $17.98 * *$ & $24.21 * *$ & $8.16 *$ \\
\hline 2. & CoPant $84212 \times$ CoSe 92423 & $-13.57 * *$ & $-19.19 * *$ & $-12.95 * *$ & -4.88 & $-13.45 * *$ & 4.06 & $9.55 *$ & -4.60 \\
\hline 3. & CoPant $98224 \times$ CoPant 97222 & 0.94 & -0.06 & -4.97 & 3.84 & -5.52 & $13.60 * *$ & $19.59 * *$ & 4.14 \\
\hline 4. & CoPant $98224 \times$ CoSe 92423 & $-6.92 *$ & $-12.38 * *$ & -5.62 & 3.13 & -6.16 & $12.82 * *$ & $18.78 * *$ & 3.43 \\
\hline 5. & CoPant $99213 \times$ CoPant 97222 & $6.95 *$ & 1.92 & 4.84 & $14.57 * *$ & 4.24 & $25.33 * *$ & $31.94 * *$ & $14.89 * *$ \\
\hline 6. & CoPant $99213 \times$ CoSe 92423 & $-8.89 * *$ & $-10.94 * *$ & -4.07 & 4.83 & -4.62 & $14.68 * *$ & $20.73 * *$ & 5.13 \\
\hline 7. & CoPant $94213 \times$ CoPant 97222 & 2.80 & -1.18 & $-7.90 *$ & 0.64 & $-8.43 *$ & $10.09 *$ & $15.90 * *$ & 0.93 \\
\hline 8. & CoPant $94213 \times$ CoSe 92423 & -2.66 & $-12.49 * *$ & -5.73 & 3.01 & -6.27 & $12.69 * *$ & $18.63 * *$ & 3.31 \\
\hline
\end{tabular}

Note : ***,***- significant at $0.5,0.01$ and 0.001 probability levels, respectively., 
Continued......

\begin{tabular}{|c|c|c|c|c|c|c|c|c|c|}
\hline \multirow[t]{3}{*}{ S.No. } & \multirow[t]{3}{*}{ CROSSES } & \multicolumn{8}{|c|}{ 4. Height } \\
\hline & & Relative heterosis & Heterobeltiosis & \multicolumn{6}{|c|}{ Standard heterosis } \\
\hline & & & & Co 1148 & Co J 64 & Co S 8436 & CoPant 3220 & Co 0238 & Co S 767 \\
\hline 1. & CoPant $84212 \times$ CoPant 97222 & $-15.24 * *$ & $-15.24 *$ & $20.11 *$ & $17.26 *$ & $43.55 * *$ & $16.04 *$ & $19.14 *$ & $33.83 * *$ \\
\hline 2. & CoPant $84212 \times$ CoSe 92423 & -7.91 & -10.00 & $33.60 * *$ & $30.43 * *$ & $59.68 * *$ & $29.07 * *$ & $32.53 * *$ & $48.87 * *$ \\
\hline 3. & CoPant $98224 \times$ CoPant 97222 & 5.53 & 0.00 & $41.70 * *$ & $38.34 * *$ & $69.35 * *$ & $36.90 * *$ & $40.56 * *$ & $57.89 * *$ \\
\hline 4. & CoPant $98224 \times$ CoSe 92423 & $-11.76 *$ & $-18.18 * *$ & $21.46 *$ & $18.58 *$ & $45.16 * *$ & $17.34 *$ & $20.48 *$ & $35.34 * *$ \\
\hline 5. & CoPant $99213 \times$ CoPant 97222 & 1.98 & -1.90 & $39.00 * *$ & $35.70 * *$ & $66.13 * *$ & $34.29 * *$ & $37.88 * *$ & $54.89 * *$ \\
\hline 6. & CoPant $99213 \times$ CoSe 92423 & -3.38 & -9.09 & $34.95 * *$ & $31.75 * *$ & $61.29 * *$ & $30.38 * *$ & $33.87 * *$ & $50.38 * *$ \\
\hline 7. & CoPant $94213 \times$ CoPant 97222 & -5.77 & -6.67 & $32.25 * *$ & $29.12 * *$ & $58.06 * *$ & $27.77 * *$ & $31.19 * *$ & $47.37 * *$ \\
\hline \multirow[t]{2}{*}{8.} & CoPant $94213 \times$ CoSe 92423 & $-11.74 *$ & $-14.55 *$ & $26.86 * *$ & $23.85 * *$ & $51.61 * *$ & $22.56 * *$ & $25.84 * *$ & $41.35 * *$ \\
\hline & & \multicolumn{8}{|c|}{ 5. Diameter } \\
\hline 1. & CoPant $84212 \times$ CoPant 97222 & 13.29 & 10.11 & 4.14 & -2.00 & -3.35 & -1.71 & -12.42 & -3.64 \\
\hline 2. & CoPant $84212 \times$ CoSe 92423 & -9.45 & $-18.75 * *$ & -3.29 & 0.00 & -10.26 & -8.73 & $-18.68 * *$ & -10.52 \\
\hline 3. & CoPant $98224 \times$ CoPant 97222 & 7.60 & 5.75 & -2.23 & -8.00 & -9.27 & -7.72 & $-17.78 * *$ & -9.54 \\
\hline 4. & CoPant $98224 \times$ CoSe 92423 & -11.56 & $-21.43 * *$ & -6.48 & -12.00 & -13.21 & -11.74 & $-21.36 * *$ & -13.47 \\
\hline 5. & CoPant $99213 \times$ CoPant 97222 & 10.71 & 10.71 & -1.17 & -7.00 & -8.28 & -6.72 & $-16.89 *$ & -8.55 \\
\hline 6. & CoPant $99213 \times$ CoSe 92423 & -8.16 & $-19.64 * *$ & -4.36 & -10.00 & -11.24 & -9.73 & $-19.57 * *$ & -11.50 \\
\hline 7. & CoPant $94213 \times$ CoPant 97222 & 2.27 & -2.17 & -4.36 & -10.00 & -11.24 & -9.73 & $-19.57 * *$ & -11.50 \\
\hline \multirow[t]{2}{*}{8.} & CoPant $94213 \times$ CoSe 92423 & $-20.59 * *$ & $-27.68 * *$ & -13.92 & $-19.00 *$ & $-20.12 * *$ & $-18.76 *$ & $-27.61 * *$ & $-20.35 * *$ \\
\hline & & \multicolumn{8}{|c|}{ 6. Single cane weight } \\
\hline 1. & CoPant $84212 \times$ CoPant 97222 & 5.15 & 4.08 & $16.97 *$ & $16.97 *$ & $20.85^{*}$ & -2.86 & -11.61 & $18.88^{*}$ \\
\hline 2. & CoPant $84212 \times$ CoSe 92423 & -5.77 & -12.5 & 12.39 & 12.39 & 16.11 & -6.67 & $-15.08^{*}$ & 14.22 \\
\hline 3. & CoPant $98224 \times$ CoPant 97222 & 5.38 & 0 & 12.39 & 12.39 & 16.11 & -6.67 & $-15.08^{*}$ & 14.22 \\
\hline 4. & CoPant $98224 \times$ CoSe 92423 & -8 & $-17.86^{* *}$ & 5.5 & 5.5 & 9 & -12.38 & $-20.28 * *$ & 7.23 \\
\hline 5. & CoPant $99213 \times$ CoPant 97222 & 2.22 & -6.12 & 5.5 & 5.5 & 9 & -12.38 & $-20.28 * *$ & 7.23 \\
\hline 6. & CoPant $99213 \times$ CoSe 92423 & -5.15 & $-17.86^{* *}$ & 5.5 & 5.5 & 9 & -12.38 & $-20.28 * *$ & 7.23 \\
\hline 7. & CoPant $94213 \times$ CoPant 97222 & 2.13 & -2.04 & 10.09 & 10.09 & 13.74 & -8.57 & $-16.81 * *$ & 11.89 \\
\hline 8. & CoPant $94213 \times$ CoSe 92423 & -10.89 & $-19.64 * *$ & 3.21 & 3.21 & 6.64 & $-14.29 *$ & $-22.01 * *$ & 4.9 \\
\hline
\end{tabular}

Note : ***,***- significant at $0.5,0.01$ and 0.001 probability levels, respectively., 
Continued......

\begin{tabular}{|c|c|c|c|c|c|c|c|c|c|}
\hline \multirow[t]{3}{*}{ S.No. } & \multirow[t]{3}{*}{ CROSSES } & \multicolumn{8}{|c|}{ 7. Brix Percent } \\
\hline & & \multirow{2}{*}{$\begin{array}{c}\text { Relative } \\
\text { heterosis }\end{array}$} & \multirow[t]{2}{*}{ Heterobeltiosis } & \multicolumn{6}{|c|}{ Standard heterosis } \\
\hline & & & & Co 1148 & Co J 64 & Co S 8436 & CoPant 3220 & Co 0238 & Co S 767 \\
\hline 1. & CoPant $84212 \times$ CoPant 97222 & $-16.12 * *$ & $-21.23 * *$ & $-20.58 * *$ & $-20.91 * *$ & $-26.19 * *$ & $-18.52 * *$ & $-25.31 * *$ & $-19.56^{* *}$ \\
\hline 2. & CoPant $84212 \times$ CoSe 92423 & $-14.62 * *$ & $-21.49 * *$ & $-17.16^{* *}$ & $-17.51 * *$ & $-23.01 * *$ & $-15.01 * *$ & $-22.1 * *$ & $-16.1 * *$ \\
\hline 3. & CoPant $98224 \times$ CoPant 97222 & -4.92 & $-8.92 *$ & $-8.17 *$ & $-8.55^{*}$ & $-14.66^{* *}$ & -5.78 & $-13.64 * *$ & -6.99 \\
\hline 4. & CoPant $98224 \times$ CoSe 92423 & $-17.16^{* *}$ & $-22.33 * *$ & $-18.05 * *$ & $-18.39 * *$ & $-23.84 * *$ & $-15.92 * *$ & $-22.93 * *$ & $-17 * *$ \\
\hline 5. & CoPant 99213 × CoPant 97222 & $-12.1 * *$ & $-14.7 * *$ & $-14 * *$ & $-14.35 * *$ & $-20.07 * *$ & $-11.76 * *$ & $-19.12 * *$ & $-12.89 * *$ \\
\hline 6. & CoPant $99213 \times$ CoSe 92423 & $-15.42 * *$ & $-19.69 * *$ & $-15.26 * *$ & $-15.62 * *$ & $-21.25 * *$ & $-13.06 * *$ & $-20.31 * *$ & $-14.18 * *$ \\
\hline 7. & CoPant $94213 \times$ CoPant 97222 & -3.75 & $-11.43 * *$ & $-10.7 * *$ & $-11.07 * *$ & $-17.01 * *$ & $-8.38 *$ & $-16.02 * *$ & $-9.56^{*}$ \\
\hline \multirow[t]{2}{*}{8.} & CoPant $94213 \times$ CoSe 92423 & -3.46 & $-12.97 * *$ & $-8.17 *$ & $-8.55^{*}$ & $-14.66^{* *}$ & -5.78 & $-13.64 * *$ & -6.99 \\
\hline & & \multicolumn{8}{|c|}{ 8. Pol Value } \\
\hline 1. & CoPant $84212 \times$ CoPant 97222 & $-17.43 * *$ & $-21.98 * *$ & $-21.72 * *$ & $-20.99 * *$ & $-30.38 * *$ & $-21.35 * *$ & $-26.78 * *$ & $-20.06^{* * *}$ \\
\hline 2. & CoPant $84212 \times$ CoSe 92423 & $-13.96 * *$ & $-19.56^{* *}$ & $-17.43 * *$ & $-16.66 * *$ & $-26.57 * *$ & $-17.05^{* *}$ & $-22.77 * *$ & $-15.68 * *$ \\
\hline 3. & CoPant $98224 \times$ CoPant 97222 & -5.85 & $-9.96^{*}$ & $-9.65^{*}$ & $-8.82 *$ & $-19.65 * *$ & $-9.24 *$ & $-15.49 * *$ & -7.74 \\
\hline 4. & CoPant $98224 \times$ CoSe 92423 & $-15.62 * *$ & $-20.17 * *$ & $-18.05 * *$ & $-17.29 * *$ & $-27.12 * *$ & $-17.67 * *$ & $-23.35 * *$ & $-16.32 * *$ \\
\hline 5. & CoPant $99213 \times$ CoPant 97222 & $-14.46^{* *}$ & $-15.64 * *$ & $-15.36^{* *}$ & $-14.57 * *$ & $-24.72 * *$ & $-14.97 * *$ & $-20.83^{* *}$ & $-13.56^{* *}$ \\
\hline 6. & CoPant $99213 \times$ CoSe 92423 & $-15.66 * *$ & $-17.74 * *$ & $-15.56 * *$ & $-14.78 * *$ & $-24.91 * *$ & $-15.17 * *$ & $-21.02 * *$ & $-13.78^{* *}$ \\
\hline 7. & CoPant $94213 \times$ CoPant 97222 & -2.8 & $-11.51 * *$ & $-11.21 * *$ & $-10.38^{*}$ & $-21.03 * *$ & $-10.8^{*}$ & $-16.95^{* *}$ & $-9.33^{*}$ \\
\hline \multirow[t]{2}{*}{8.} & CoPant $94213 \times$ CoSe 92423 & -0.8 & $-10.61 * *$ & $-8.24 *$ & -7.38 & $-18.39 * *$ & -7.81 & $-14.17 * *$ & -6.29 \\
\hline & & \multicolumn{8}{|c|}{ 9. Sucrose Percent } \\
\hline 1. & CoPant $84212 \times$ CoPant 97222 & $-16.19 * *$ & $-20.4 * *$ & $-20.16^{* *}$ & $-19.37 * *$ & $-28.56^{* *}$ & $-19.78 * *$ & $-24.93 * *$ & $-18.52 * *$ \\
\hline 2. & CoPant $84212 \times$ CoSe 92423 & $-12.79 * *$ & $-18 * *$ & $-15.98 * *$ & $-15.15 * *$ & $-24.82 * *$ & $-15.58 * *$ & $-21 * *$ & $-14.25 * *$ \\
\hline 3. & CoPant $98224 \times$ CoPant 97222 & -5.09 & $-8.91 *$ & $-8.63^{*}$ & $-7.73 *$ & $-18.25 * *$ & $-8.2 *$ & $-14.09 * *$ & -6.75 \\
\hline 4. & CoPant $98224 \times$ CoSe 92423 & $-14.43^{* *}$ & $-18.71 * *$ & $-16.7 * *$ & $-15.88^{* *}$ & $-25.47 * *$ & $-16.3^{* * *}$ & $-21.68 * *$ & $-14.99 * *$ \\
\hline 5. & CoPant $99213 \times$ CoPant 97222 & $-13.58^{* *}$ & $-14.51^{* *}$ & $-14.25^{* *}$ & $-13.4 * *$ & $-23.28 * *$ & $-13.84 * *$ & $-19.38^{* *}$ & $-12.49 * *$ \\
\hline 6. & CoPant $99213 \times$ CoSe 92423 & $-14.51 * *$ & $-16.32 * *$ & $-14.25 * *$ & $-13.4 * *$ & $-23.28 * *$ & $-13.84 * *$ & $-19.38 * *$ & $-12.49 * *$ \\
\hline 7. & CoPant $94213 \times$ CoPant 97222 & -2.19 & $-10.34 * *$ & $-10.07^{*}$ & $-9.18 *$ & $-19.54 * *$ & $-9.64 *$ & $-15.45^{* *}$ & $-8.22 *$ \\
\hline 8. & CoPant $94213 \times$ CoSe 92423 & -0.39 & $-9.56^{*}$ & -7.34 & -6.42 & $-17.09 * *$ & -6.89 & $-12.87 * *$ & -5.43 \\
\hline
\end{tabular}

Note : ***,***- significant at $0.5,0.01$ and 0.001 probability levels, respectively., 
Continued........

\begin{tabular}{|c|c|c|c|c|c|c|c|c|c|}
\hline \multirow[t]{3}{*}{ S.No. } & \multirow[t]{3}{*}{ CROSSES } & \multicolumn{8}{|c|}{ 10. Purity Percent } \\
\hline & & \multirow[t]{2}{*}{ Relative heterosis } & \multirow[t]{2}{*}{ Heterobeltiosis } & \multicolumn{6}{|c|}{ Standard heterosis } \\
\hline & & & & Co 1148 & Co J 64 & Co S 8436 & CoPant 3220 & Co 0238 & Co S 767 \\
\hline 1. & CoPant $84212 \times$ CoPant 97222 & -0.51 & -1.56 & 0.79 & 2.08 & $-3.16^{*}$ & -1.64 & 0.51 & 1.44 \\
\hline 2. & CoPant $84212 \times$ CoSe 92423 & 0.85 & -0.75 & 1.62 & 2.92* & -2.36 & -0.83 & 1.33 & 2.27 \\
\hline 3. & CoPant $98224 \times$ CoPant 97222 & -0.41 & -0.48 & -0.12 & 1.16 & $-4.03 * *$ & -2.53 & -0.4 & 0.52 \\
\hline 4. & CoPant $98224 \times$ CoSe 92423 & 1.78 & 1.16 & 1.54 & 2.83 & -2.44 & -0.92 & 1.25 & 2.19 \\
\hline 5. & CoPant $99213 \times$ CoPant 97222 & -2 & $-3.73 *$ & 0.03 & 1.3 & $-3.89 * *$ & -2.39 & -0.26 & 0.67 \\
\hline 6. & CoPant $99213 \times$ CoSe 92423 & -0.72 & $-2.99 *$ & 0.79 & 2.08 & $-3.16^{*}$ & -1.64 & 0.51 & 1.44 \\
\hline 7. & CoPant $94213 \times$ CoPant 97222 & 1.1 & 0.48 & 0.71 & 2 & $-3.24 *$ & -1.72 & 0.42 & 1.36 \\
\hline \multirow[t]{2}{*}{8.} & CoPant $94213 \times$ CoSe 92423 & 1.8 & 1.72 & 0.85 & 2.14 & $-3.1^{*}$ & -1.58 & 0.57 & 1.5 \\
\hline & & \multicolumn{8}{|c|}{ 11. C.C.S. Percent } \\
\hline 1. & CoPant $84212 \times$ CoPant 97222 & $-20.46^{* *}$ & $-23.71 * *$ & $-24.89 * *$ & $-26.63 * *$ & $-24.49 * *$ & $-23.43 * *$ & $-25.72 * *$ & $-24.17 * *$ \\
\hline 2. & CoPant $84212 \times$ CoSe 92423 & $-17.73 * *$ & $-22.81 * *$ & $-20.38 * *$ & $-22.22 * *$ & $-19.95 * *$ & $-18.83 * *$ & $-21.26^{* *}$ & $-19.62 * *$ \\
\hline 3. & CoPant $98224 \times$ CoPant 97222 & $-10.17 * *$ & $-12.95 * *$ & $-14.3 * *$ & $-16.28 * *$ & $-13.84 * *$ & $-12.63 * *$ & $-15.24 * *$ & $-13.48 * *$ \\
\hline 4. & CoPant $98224 \times$ CoSe 92423 & $-19.56 * *$ & $-23.76 * *$ & $-21.36 * *$ & $-23.18 * *$ & $-20.94 * *$ & $-19.83 * *$ & $-22.23 * *$ & $-20.61 * *$ \\
\hline 5. & CoPant $99213 \times$ CoPant 97222 & $-16.29 * *$ & $-18.13 * *$ & $-19.4 * *$ & $-21.26 * *$ & $-18.97 * *$ & $-17.83 * *$ & $-20.29 * *$ & $-18.63 * *$ \\
\hline 6. & CoPant $99213 \times$ CoSe 92423 & $-17.89 * *$ & $-21.48 * *$ & $-19 * *$ & $-20.88 * *$ & $-18.57 * *$ & $-17.43 * *$ & $-19.9 * *$ & $-18.23 * *$ \\
\hline 7. & CoPant $94213 \times$ CoPant 97222 & -4.11 & $-13.94 * *$ & $-15.28 * *$ & $-17.24 * *$ & $-14.83 * *$ & $-13.63 * *$ & $-16.21 * *$ & $-14.47 * *$ \\
\hline \multirow[t]{2}{*}{8.} & CoPant $94213 \times$ CoSe 92423 & -3.57 & $-15.21 * *$ & $-12.53 * *$ & $-14.56 * *$ & $-12.07 * *$ & $-10.84 * *$ & $-13.5^{* *}$ & $-11.7 * *$ \\
\hline & & \multicolumn{8}{|c|}{ 12. Cane yield } \\
\hline 1. & CoPant $84212 \times$ CoPant 97222 & 2.62 & 2.36 & 7.33 & $16.73 *$ & 9.99 & 6.18 & 8.69 & 21.1* \\
\hline 2. & CoPant $84212 \times$ CoSe 92423 & $-13.19^{*}$ & $-17.94 * *$ & -3.88 & 4.54 & -1.5 & -4.91 & -2.66 & 8.45 \\
\hline 3. & CoPant $98224 \times$ CoPant 97222 & 0.3 & -4.39 & 0.25 & 9.04 & 2.74 & -0.82 & 1.53 & 13.12 \\
\hline 4. & CoPant $98224 \times$ CoSe 92423 & -6.56 & $-15.36^{*}$ & -0.87 & 7.82 & 1.59 & -1.92 & 0.4 & 11.86 \\
\hline 5. & CoPant $99213 \times$ CoPant 97222 & 7.71 & 3.75 & 8.78 & $18.31 *$ & 11.48 & 7.62 & 10.17 & $22.74 * *$ \\
\hline 6. & CoPant $99213 \times$ CoSe 92423 & -5.72 & $-13.77 *$ & 1.01 & 9.86 & 3.51 & -0.07 & 2.29 & 13.97 \\
\hline 7. & CoPant $94213 \times$ CoPant 97222 & 1.4 & -3.2 & 1.5 & 10.39 & 4.02 & 0.42 & 2.79 & 14.53 \\
\hline 8. & CoPant $94213 \times$ CoSe 92423 & -2.84 & -11.88 & 3.22 & 12.26 & 5.78 & 2.11 & 4.53 & $16.46^{*}$ \\
\hline
\end{tabular}

Note : *,*****- significant at $0.5,0.01$ and 0.001 probability levels, respectively., 
Continued......

\begin{tabular}{|c|c|c|c|c|c|c|c|c|c|}
\hline \multirow[t]{3}{*}{ S.No. } & \multirow[t]{3}{*}{ CROSSES } & \multicolumn{8}{|c|}{ 13. C.C.S. yield } \\
\hline & & \multirow[t]{2}{*}{ Relative heterosis } & \multirow[t]{2}{*}{ Heterobeltiosis } & \multicolumn{6}{|c|}{ Standard heterosis } \\
\hline & & & & Co 1148 & Co J 64 & Co S 8436 & CoPant 3220 & Co 0238 & Co S 767 \\
\hline 1. & CoPant $84212 \times$ CoPant 97222 & $-18.67 * *$ & $-22.07 * *$ & $-20.1^{* *}$ & $-15.93^{*}$ & $-16.56^{*}$ & $-19.72 *$ & $-20.03 * *$ & -9.32 \\
\hline 2. & CoPant $84212 \times$ CoSe 92423 & $-25.2 * *$ & $-30.61 * *$ & $-23.77 * *$ & $-19.79 *$ & $-20.39^{*}$ & $-23.41 * *$ & $-23.7 * *$ & -13.49 \\
\hline 3. & CoPant $98224 \times$ CoPant 97222 & -9.64 & $-16.1^{*}$ & -13.98 & -9.5 & -10.17 & -13.58 & -13.91 & -2.38 \\
\hline 4. & CoPant $98224 \times$ CoSe 92423 & $-21.24 * *$ & $-29.13 * *$ & $-22.14^{* *}$ & $-18.08 *$ & $-18.69^{*}$ & $-21.77 * *$ & $-22.07 * *$ & -11.64 \\
\hline 5. & CoPant $99213 \times$ CoPant 97222 & -9.89 & $-14.91 *$ & -12.76 & -8.21 & -8.9 & -12.35 & -12.69 & -0.99 \\
\hline 6. & CoPant $99213 \times$ CoSe 92423 & $-18.46^{* *}$ & $-25.42 * *$ & $-18.06^{*}$ & -13.79 & -14.43 & $-17.67 *$ & $-17.99 *$ & -7.01 \\
\hline 7. & CoPant $94213 \times$ CoPant 97222 & -3.11 & $-16.5^{*}$ & -14.39 & -9.93 & -10.6 & -13.99 & -14.32 & -2.85 \\
\hline 8. & CoPant $94213 \times$ CoSe 92423 & -2.1 & $-18^{* *}$ & -9.91 & -5.21 & -5.92 & -9.48 & -9.83 & 2.24 \\
\hline
\end{tabular}

Note : *,*****- significant at $0.5,0.01$ and 0.001 probability levels, respectively.,

Table. 3 Best crosses identified on the basis of heterosis for different characters in sugarcane

\begin{tabular}{|c|c|c|c|c|c|c|c|c|}
\hline & \multicolumn{8}{|c|}{ Estimation of heterosis } \\
\hline & \multirow[t]{2}{*}{ Relative heterosis } & \multirow[t]{2}{*}{ Heterobeltiosis } & \multicolumn{6}{|l|}{ Standard heterosis } \\
\hline & & & Co 1148 & Co J 64 & Co S 8436 & CoPant 3220 & Co 0238 & Co S 767 \\
\hline Germination & & & $\mathrm{L} 1 \times \mathrm{T} 1$ & $\mathrm{~L} 1 \times \mathrm{T} 1, \mathrm{~L} 3 \times \mathrm{T} 2$ & $\mathrm{~L} 1 \times \mathrm{T} 1, \mathrm{~L} 3 \times \mathrm{T} 2$ & $\mathrm{~L} 1 \times \mathrm{T} 1, \mathrm{L3} \times \mathrm{T} 2$ & $\mathrm{~L} 1 \times \mathrm{T} 1, \mathrm{~L} 3 \times \mathrm{T} 2$ & $\mathrm{~L} 1 \times \mathrm{T} 1, \mathrm{~L} 3 \times \mathrm{T} 2$ \\
\hline Tillers & $\mathrm{L} 1 \times \mathrm{T} 1, \mathrm{~L} 2 \times \mathrm{T} 1$ & L1 $\times$ T1, & $\begin{array}{l}\mathrm{L} 1 \times \mathrm{T} 1, \mathrm{~L} 2 \times \mathrm{T} 1, \\
\mathrm{~L} 3 \times \mathrm{T} 1, \mathrm{~L} 3 \times \mathrm{T} 2\end{array}$ & $\begin{array}{l}\mathrm{L} 1 \times \mathrm{T} 1, \mathrm{~L} 2 \times \mathrm{T} 1 \\
\mathrm{~L} 3 \times \mathrm{T} 1\end{array}$ & L1 $\times$ T1 & $\begin{array}{l}\mathrm{L} 1 \times \mathrm{T} 1, \mathrm{~L} 2 \times \mathrm{T} 1 \\
\mathrm{~L} 2 \times \mathrm{T} 2, \mathrm{~L} 3 \times \mathrm{T} 1 \\
\mathrm{~L} 4 \times \mathrm{T} 1\end{array}$ & $\mathrm{~L} 1 \times \mathrm{T} 1, \mathrm{~L} 3 \times \mathrm{T} 1$ & $\begin{array}{l}\text { L1× T1, } \\
\text { L2 } \times \text { T1, L3× T1 }\end{array}$ \\
\hline NMC & $\mathbf{L 3} \times \mathbf{T} 1$ & & & $\mathrm{~L} 1 \times \mathrm{T} 1, \mathrm{~L} 3 \times \mathrm{T} 1$ & & $\begin{array}{l}\mathrm{L} 1 \times \mathrm{T} 1, \mathrm{~L} 2 \times \mathrm{T} 1 \\
\mathrm{~L} 2 \times \mathrm{T} 2, \mathrm{~L} 3 \times \mathrm{T} 1 \\
\mathrm{~L} 3 \times \mathrm{T} 2, \mathrm{~L} 4 \times \mathrm{T} 1 \\
\mathrm{~L} 4 \times \mathrm{T} 2\end{array}$ & $\begin{array}{l}\mathrm{L} 1 \times \mathrm{T} 2, \mathrm{~L} 1 \times \mathrm{T} 1, \\
\mathrm{~L} 2 \times \mathrm{T} 1, \mathrm{~L} 2 \times \mathrm{T} 2, \\
\mathrm{~L} 3 \times \mathrm{T} 1, \mathrm{L3} \times \mathrm{T} 2, \\
\mathrm{~L} 4 \times \mathrm{T} 1, \mathrm{~L} 4 \times \mathrm{T} 2\end{array}$ & L1× T1, L3× T1 \\
\hline Height & & & $\begin{array}{l}\mathrm{L} 1 \times \mathrm{T} 2, \mathrm{~L} 1 \times \mathrm{T} 1, \mathrm{~L} 2 \times \mathrm{T} 1, \\
\mathrm{~L} 2 \times \mathrm{T} 2, \mathrm{~L} 3 \times \mathrm{T} 1, \mathrm{~L} 3 \times \mathrm{T} 2 \\
\mathrm{~L} 4 \times \mathrm{T} 1, \mathrm{~L} 4 \times \mathrm{T} 2\end{array}$ & $\begin{array}{l}\mathrm{L} 1 \times \mathrm{T} 1, \mathrm{~L} 1 \times \mathrm{T} 2 \\
\mathrm{~L} 2 \times \mathrm{T} 1, \mathrm{~L} 2 \times \mathrm{T} 2 \\
\mathrm{~L} 3 \times \mathrm{T} 1, \mathrm{~L} 3 \times \mathrm{T} 2 \\
\mathrm{~L} 4 \times \mathrm{T} 1, \mathrm{~L} 4 \times \mathrm{T} 2\end{array}$ & $\begin{array}{l}\mathrm{L} 1 \times \mathrm{T} 1, \mathrm{~L} 1 \times \mathrm{T} 2 \\
\mathrm{~L} 2 \times \mathrm{T} 1, \mathrm{~L} 2 \times \mathrm{T} 2 \\
\mathrm{~L} 3 \times \mathrm{T} 1, \mathrm{L3} \times \mathrm{T} 2 \\
\mathrm{~L} 4 \times \mathrm{T} 1, \mathrm{~L} 4 \times \mathrm{T} 2\end{array}$ & $\begin{array}{l}\mathrm{L} 1 \times \mathrm{T} 1, \mathrm{~L} 1 \times \mathrm{T} 2 \\
\mathrm{~L} 2 \times \mathrm{T} 1, \mathrm{~L} 2 \times \mathrm{T} 2 \\
\mathrm{~L} 3 \times \mathrm{T} 1, \mathrm{L3} \times \mathrm{T} 2 \\
\mathrm{~L} 4 \times \mathrm{T} 1, \mathrm{~L} 4 \times \mathrm{T} 2\end{array}$ & $\begin{array}{l}\mathrm{L} 1 \times \mathrm{T} 1, \mathrm{~L} 1 \times \mathrm{T} 2 \\
\mathrm{~L} 2 \times \mathrm{T} 1, \mathrm{~L} 2 \times \mathrm{T} 2 \\
\mathrm{~L} 3 \times \mathrm{T} 1, \mathrm{L3} \times \mathrm{T} 2 \\
\mathrm{~L} 4 \times \mathrm{T} 1, \mathrm{~L} 4 \times \mathrm{T} 2\end{array}$ & $\begin{array}{l}\mathrm{L} 1 \times \mathrm{T} 1, \mathrm{~L} 1 \times \mathrm{T} 2, \\
\mathrm{~L} 2 \times \mathrm{T} 1, \mathrm{~L} 2 \times \mathrm{T} 2, \\
\mathrm{~L} 3 \times \mathrm{T} 1, \mathrm{~L} 3 \times \mathrm{T} 2, \\
\mathrm{~L} 4 \times \mathrm{T} 1, \mathrm{~L} 4 \times \mathrm{T} 2\end{array}$ \\
\hline \multicolumn{9}{|l|}{ Diameter } \\
\hline $\begin{array}{l}\text { Single cane } \\
\text { weight }\end{array}$ & & & L1×T1 & L1×T1 & L1×T1 & & & L1×T1 \\
\hline \multicolumn{9}{|l|}{ Brix } \\
\hline \multicolumn{9}{|l|}{ Pol } \\
\hline \multicolumn{9}{|l|}{ Sugar } \\
\hline \multicolumn{9}{|l|}{ Purity \% } \\
\hline \multicolumn{9}{|l|}{ CCS \% } \\
\hline Cane yield & & & & $\mathrm{L} 1 \times \mathrm{T} 1, \mathrm{~L} 3 \times \mathrm{T} 1$ & & & & $\begin{array}{l}\mathrm{L} 1 \times \mathrm{T} 1, \mathrm{~L} 3 \times \mathrm{T} 1, \\
\mathrm{~L} 4 \times \mathrm{T} 2\end{array}$ \\
\hline CCS yield & & & & & & & & \\
\hline
\end{tabular}

Notation:-

L1= CoPant 84212, L2=CoPant 98224, L3=CoPant 99213, L4= CoPant 94213, T1=CoPant 97222, T2= CoSe 92423 
While exhibited significant variation for the characters like Cane Height $\left(0.087^{*}\right)$, Cane Diameter $(0.137 *)$ and Cane yield $(136.437 *)$ among the cross. This indicates that genetic material was suitable for determining general and specific combining ability of parents and the crosses which required for heterosis estimation. The variability studies by analysis of variance however, represent a rough estimate of the variation present in the material.

For the development of hybrids it is important that a hybrid should manifest a high magnitude of heterosis for its commercial exploitation. Sugarcane is polyploid and highly heterozygous thereby high variability is expected in F1s. Since sugarcane is a vegetatively propagated crop, heterosis can be fixed and exploited in F1 generation. Heterosis estimates are presented for thirteen characters in the Table 2. Results revealed that positive and significant relative heterosis and heterobeltiosis for tillers count was exhibited by hybrid CoPant $84212 \times$ CoPant 97222 and for number of number of millable canes by CoPant $99213 \times$ CoPant 97222. Hybrid CoPant $84212 \times$ CoPant 97222 was recorded with significant positive economic heterosis for germination Percent, tillers count and single cane weight over checks Co 1148, Co J 64, Co S 8436, Co S 767 and for germination Percent and tillers count over checks CoPant 3220 and Co 0238. Hybrid CoPant $98224 \times$ CoPant 97222 exhibited significant positive economic heterosis for cane height over all the six check varieties. Another hybrid, CoPant $99213 \times$ CoPant 97222 gave significant positive economic heterosis for number of millable canes and cane yield over two checks Co J 64 and Co S 767 and only for number of millable canes over checks CoPant 3220 and Co 0238. Cross, CoPant $84212 \times$ CoSe 92423 exhibited positive and significant economic heterosis for purity Percent over check Co J 64. Heterotic response along-with per se performance should be taken into consideration for the selection of parental combination for hybridization (Katiyar, 1979). Crosses involved one of the parents with high per se performance (CoPant 84212, CoPant
98224 and CoPant 97222) gave high significant positive heterosis for tillers count, number of millable canes, germination Percent, single cane weight, cane height and purity Percent. However, in one cross combination CoPant $84212 \times$ CoPant 97222 exhibited high positive relative heterosis and heterobeltiosis for tillers count and high economic heterosis for germination Percent, tillers count and single cane weight, high $\mathrm{x}$ high per se performance were also responsible for high heterosis, indicating additive $\mathrm{x}$ additive type of gene interaction was involved. Yang and Chu, (1962) also reported similar results for most of the characters in sugarcane (Table 3).

From the present study it can be concluded that genetic variability exists among the studied genotypes for all the traits. Involving the genotypes from different heterotic groups in crossing program often leads to heterosis and yield stability of the new cultivars. Therefore, from the present investigation it may be concluded that the hybrids CoPant $84212 \times$ CoPant 97222, CoPant $99213 \times$ CoPant 97222 and CoPant $98224 \times$ CoPant 97222 can be identified as best cross combinations and can be exploited for the improvement of various traits viz., Germination Percentage, tillers count, Number of Millable Cane, Cane Height, Single Cane Weight and Cane Yield potential in sugarcane.

\section{References}

Alarmelu, S., Hemaprabha, G., Nagarajan, R. and Shanthi, R. M., 2010. Combining ability for yield and quality in Sugarcane. Electronic Journal of Plant Breeding. 1(4): 742-746.

Alexander Pswarayi, A. and Vivek, B.S. 2008. Combining ability amongst CIMMYT's early maturing maize (Zea mays L.) germplasm under stress and non-stress conditions and identify cation of testers. Euphytica, 162: 353-362.

Anbanandan, V., Eswaran, R. and Sabesan, T. 2017. 'Heterosis In Interspecific And Intergeneric Progenies Of Sugarcane' Life 
Sciences International Research Journal, 4(1): 43-46.

Comstock, R. E. and Robinson, H. F. 1948. The components of genetic variance in populations of biparental progenies and their uses in estimating the average degree of dominance. Biometrics, 4: 254266.

Da-Costa, M.L.M., Amorim, L.L.B., Onofre, A.V.C., De-Melo, L. and De-Oliveira, M.B.M. 2011. Assessment of Genetic Diversity In Contrasting Sugarcane Varieties Using Inter-Simple Sequence Repeat (Issr) Markers. American Journal of Plant Sciences, 2: 425-432.

Gomez, K. A. and Gomez, A. A. 1984. Statistical Procedures for Agricultural Research. $2^{\text {nd }}$ Edn. A Wiley-Interscience Publication, New York.

Hayman, B.I. 1958. The theory and analysis of diallel crosses. II. Genetics. 43, pp 63 65 .

India. Ministry of Agriculture and Farmers Welfare, Department of Agriculture, Cooperation and Farmers Welfare. 2016. Agriculture Statistics at a Glance. Delhi. Controller of Publication. 489p

Katiyar, R.P. 1979. Heterosis in relation to per se performance and effects of general combining ability in chickpea. Indian $J$. Agric. Sci., 49(5): 313-7.

Loganathan, P., Saravanan K. and Ganesan, J. 2001. Heterosis for yield and yield components in greengram. Legume Res., 24: 77-81.

Menossi, M., Silva-Filho, M.C., Vincentz, M., Van-Sluys, M.A., Souza, G.M. 2008.
Sugarcane Functional: Gene Discovery For Agronomic Trait Development. International Journal of Plant Genomics, 4: 1-11.

Patil, P.P. and Patel, D.U. 2017. Study of Genetic Variability and Heritability in Sugarcane. International Journal of Current Microbiology and Applied Sciences, 6(9): 3112-3117.

Rajeswari, S., Anbanandan, V., Shanmugasundaram, K., Thirugnanakumar, S. and Krishnamurthi, M. Wide hybridization and exploitation of heterosis in sugarcane (Saccharum officinarum L.). In: National Seminar on hybrid breeding in crop plants held on 3-4 March, 2004 at Annamalai University, Annamalainagar, India.

Shull, G.F. 1952. Beginnings of the heterosis concept. In Heterosis (Gowen, J.W., ed.), pp. 14-48, Iowa State College Press.

Singh, R. K. and Chaudhary, B. D. 1985. Analysis in Biometrical Genetics. Kalyani Publishers, New Delhi.

Tyagi, A. P. and Lal, P. 2005. 'Line x tester analysis in sugarcane (Saccharum officinarum)', The South Pacific Journal of Natural Science, 23: 30-36.

Verma, P.S. and Singh, S.B. 2004. Heterosis in Relation to Per se Performance and Effects of General Combining Ability in Sugarcane. Sugar Tech., 6(3): 181-185.

Verma, P.S. 1990. Heterosis in sugarcane. Indian J. Genet., 50(2): 117-120.

Yang, T.C. and Chu, C.C. 1962. Evaluation of combining ability in sugarcane (Part-1) report of Taiwan. Sug. Exp. Sta., 26: 1-10.

\section{How to cite this article:}

Deepankar Pandey, S.P. Singh, A.S. Jeena and Tabassum. 2019. Line $\times$ Tester Analysis to Determine Extent of Heterosis for Various Yield and Quality Parameters in Sugarcane (Saccharum officinarum). Int.J.Curr.Microbiol.App.Sci. 8(03): 1537-1546.

doi: https://doi.org/10.20546/ijcmas.2019.803.177 